\title{
Volvulus of Caecum
}

\section{Abdul Ghafoor D alwani, A bdul Razaque Shaikh and Gulshan Ara Shaikh}

\begin{abstract}
This case report describes volvulus of caecum in a young boy of 18 years, who presented with symptoms of acute abdomen. The condition was diagnosed at laparotomy and the patient was treated with right hemicolectomy.
\end{abstract}

KEY WORDS: Volvulus of Caecum. Right hemicolectomy. Acute abdomen. Labarotomy.

\section{INTRODUCTION}

Acute volvulus of the caecum is a surgical emergency. Colonic volvulus represents $10 \%$ of cases of largebowel obstruction, and cecal volvulus represents approximately $40 \%$ of all such cases ${ }^{1,}$ The cecal volvulus accounts for $1 \%$ of mechanical bowel obstruction and $25-40 \%$ cases of colonic volvulus. Sigmoid volvulus is by far the most common, accounting for about $65 \%$ of all cases of colonic volvulus. Cecal volvulus usually occurs clockwise around the ileocecal vessels and involves the terminal portion of ileum and ascending colon. It is a closed loop obstruction that may lead to vascular compromise with consequent gangrene and perforation.

\section{CASE REPORT}

An 18 year old boy presented in emergency ward with severe pain in right iliac fossa for last two days. On examination tenderness was present in the right iliac fossa, patient looked ill and restless. He also gave history of off and on localized distension in right iliac fossa, which was relieved spontaneously followed by loose motions. There was no history of vomiting, constipation and malena. TLC count was increased and plain abdominal X-ray showed distended gas filled bowel loops in the center of abdomen (Figure I). Provisional diagnosis of acute appendicitis was made. Exploratory laparotomy through right lower paramedian incision was done. On exploration, caecum was found to be twisted with a gangrenous spot on its surface. (Figure II) Caecum was rotated clockwise, involving the terminal portion of ileum also compromising its viability. After confirming the non viability of the bowel loop, resection of caecum, ascending and terminal portion of ileum was performed and ileotransverse colostomy was done. Postoperative recovery was smooth and uneventful.

\section{DISCUSSION}

In Cecal volvulus the caecum is mobile and shares a common mesentery with the ileum. It is free to rotate, usually clockwise, out of the right iliac fossa to the mid or left side of the upper abdomen, producing a closed loop obstruction of the ascending colon and distal ileum. Perforation results in a life threatening complication associated with mortality of $20 \%{ }^{2}$. When associ-
FIGURE I:

X-RAY SHOWED DISTENDED GAS FILLED BOWEL LOOPS IN THE CENTER OF ABDOMEN

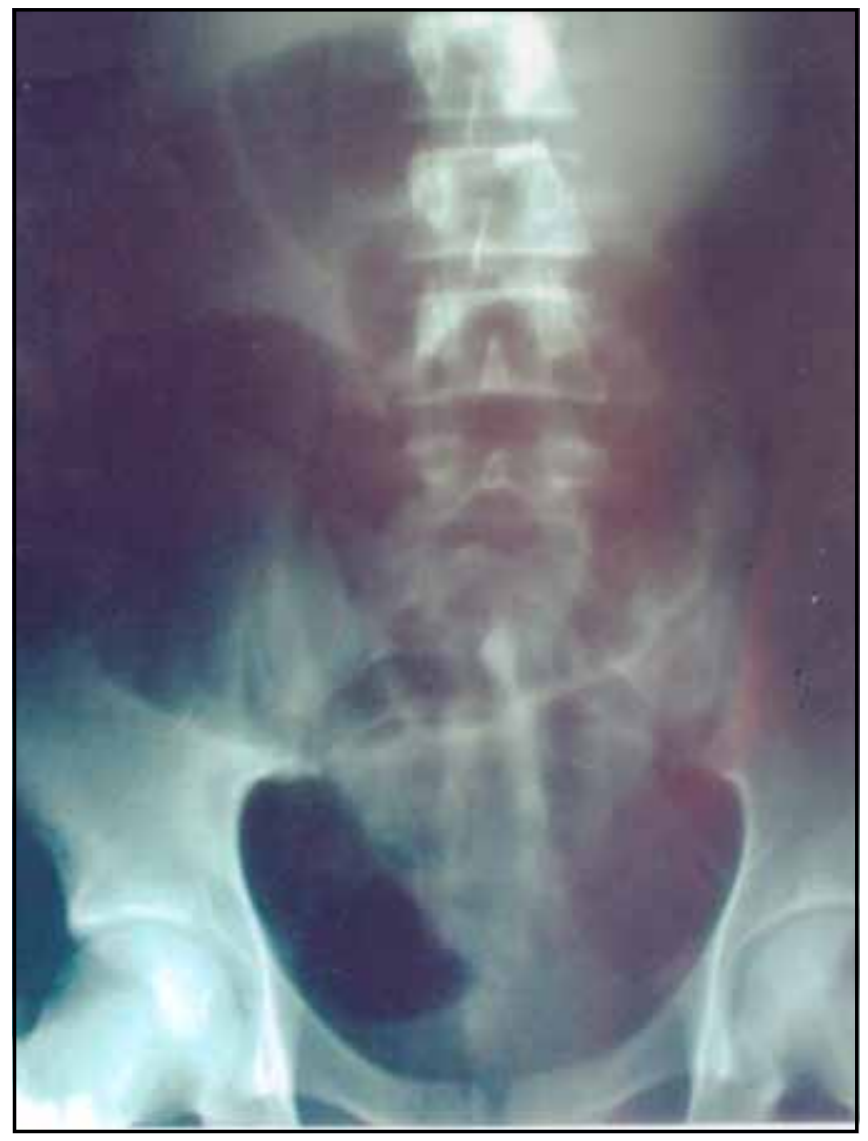

ated with cecal gangrene, the post operative mortality is reported to be as high as $45 \%{ }^{3}$. Cecal volvulus may present as acute abdomen with intestinal strangulation secondary to mesenteric torsion or with features of intestinal obstruction. The peak age of incidence is 30 to 40 years. Cecal volvulus occurs predominantly in patients with poor right colon fixation and affects approximately $10 \%$ to $25 \%{ }^{4}$ of population. Such poor fixation leads to excessive cecal mobility and the potential for vascular compromise, which may occur as a result of intestinal dilation and vascular torsion. Torsion of the caecum takes place for a num- 
FIGURE II:

\section{ON EXPLORATION CAECUM WAS FOUND TO BE TWISTED WITH A GANGRENOUS SPOT ON ITS SURFACE}

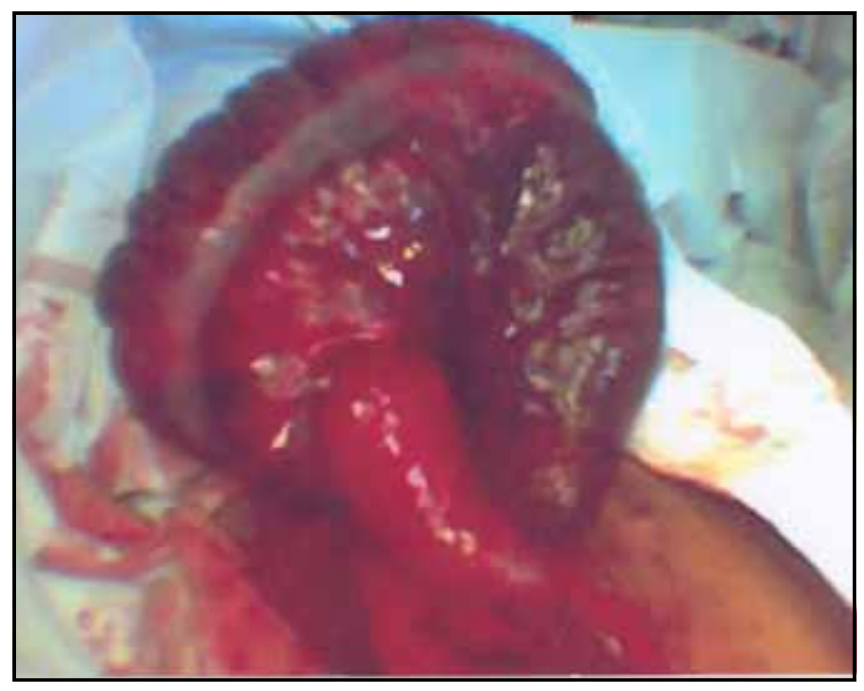

ber of reasons: sudden distension of the caecum by trauma, pressure, constipation, or a distal colonic obstruction $^{2,3,5}$. There often appears to have a triggering event, such as recent laparotomy. It is also well recognized following gynecological procedures. The presenting symptoms are usually non specific. Abdominal pain is almost invariably present. Nausea, vomiting, constipation \& distension occur in about one third of patients. In thin patients it may be possible to palpate the resonant distended caecum in the central or upper abdomen while the right iliac fossa is empty. The key to the diagnosis of cecal volvulus is plain abdominal radiograph. The caecum appears as gas filled comma shaped bowel facing inferiorly \& to the right. The diagnosis is made in 50 percent of patients. Barium enema may reveal complete obstruction to flow with a characteristic "Bird beak" appearance at the base of the torsion. It may also be useful to exclude any other predisposing colonic lesion and sometimes it has been effective in reducing the volvulus ${ }^{6}$. The management of patients who present with cecal volvulus without evidence of strangulation is fixation of the caecum (cecopexy).Cecopexy provides a safe alternative to resection and primary anastomosis in suitably selec- tive patients ${ }^{7}$. This is best achieved by combining suture fixation to the lateral abdominal wall with stripping of the parietal \& visceral peritoneum to create raw surface that will stick firmly and permanently together. The addition of a tube caecostomy has been advocated in addition to cecopexy and this has been shown to reduce the recurrence rate. The cecostomy tube can be removed after 1 week and the tract will normally close spontaneously within a few days. In severely ill patients, it may be expedient to exteriorize the proximal and distal bowel ends following resection, as an ileostomy and mucous fistula. These can then be anastomosed in second operation 6 weeks later ${ }^{7}$. Endoscopic deflation has little place but there is a chance of recurrence. The definitive treatment of cecal volvulus even when the bowel is viable is right hemicolectomy, as it avoids all risk of recurrence and mortality is lower than that obtained with conservative treatment ${ }^{8,9}$.

\section{REFERENCE}

1. Nelson TG, Bowers WF. Volvulus of the caecum and sigmoid colon: An analysis of nine cases. Arch Surg1956; 72(3):469-78

2. Johnson CD, Rice RP, Kelvin FM. The radiological evaluation of gross cecal distension. Emphasis on cecal ileus. AJR Am J Roentgenol 1985; 145 (6):1211-7

3. Tejler G, Jibron $\mathrm{H}$. Volvulus of the caecum. Report of 26 cases and review of the literature. Dis Colon Rect 1988; 31(6):445-9

4. Rogers RL, Harford FJ. Mobile caecum syndrome. Dis Colon Rect.1984; 27(6):399-402

5. Smith WR, Goodwin JN. Cecal volvulus. Am J Surg 1973; 126(2):215-22

6. Madiba TE, Thomson SR. The management of cecal volvulus. Dis Colon Rectum 2002; 45 (2):264-7

7. Grossman EM, Johnson FE, Enger KT. Ceacal volvulus: outcome of management by celiotomy. Tech Coloproctol 1999; 3(3):139-43

8. Tuech JJ, Pessaux P, Regenet N. Results of resection for volvulus of the right colon. Tech Coloproctol 2002; 6(2):97-9

9. Majeski J. Operative therapy for cecal volvulus combining resection with colopexy. Am J Surg 2005; 189(2):211-3.

\section{AUTHOR AFFILIATION:}

Dr. Abdul Ghafoor Dalwani (Corresponding Author) Department of General Surgery

Liaquat University of Medical and Health Sciences, (LUMHS) Jamshoro, Sindh-Pakistan.

Emial: aghafoordelwani@yahoo.com

\section{Prof. Abdul Razaque Shaikh \\ Department of Surgery \\ LUMHS Jamshoro, Sindh-Pakistan.}

Dr. Gulshan Ara Shaikh

Department of Radiology

ISRA University Hala Road, Hyderabad, Sindh-Pakistan. 